\title{
Ethoxycarbonylation of 3-keto-4-methyl-5-oxacephams
}

\author{
Arkadiusz Kazimierski, Zbigniew Kałuża, and Marek Chmielewski* \\ Institute of Organic Chemistry PAN, Kasprzaka 44/52, 01-224 Warsaw, Poland \\ E-mail: chmiel@icho.edu.pl
}

Dedicated to Professor Mieczysław Mąkosza on his $\mathbf{7 0}^{\text {th }}$ birthday

(received 11 Nov 03; accepted 13 Apr 04; published on the web 16 Apr 04)

\begin{abstract}
Due to their multifunctional character, the ethoxycarbonylation of 3-keto-5-oxa-cephams (10, 27 and 28) using ethyl cyanoformate in THF/HMPA solution led to expected products 14, 27 and 29 in a low yield only. Apart from the desired compounds, the $O$-acylation, $C$-alkylation at $\mathrm{C}$-7, and the addition of $\mathrm{CN}^{-}$anion to the keto group were also observed.
\end{abstract}

Keywords: 5-Oxacephams, alkoxycarbonylation

\section{Introduction}

Synthesis of the racemic 5-oxacephalotin (1) and 5-oxacephamandol (2) by Merck scientists ${ }^{1,2}$ has shown that the substitution of cephalosporin sulfur atom by the oxygen atom resulted in the same or higher antibiotic activity in comparison to the parent drug. A thorough investigation undertaken during late seventies by Shionogi Co. ${ }^{3}$ led to the introduction to the market of two novel oxacephamycins: latamoxef (3) and flomoxef (4) that represent third and fourth generation of this class of antibiotics. At the end of the eighties the Merck and Meiji groups ${ }^{4}$ reported on a new 4-methyl-cephalosporin 5 which offers the stability toward $\beta$-lactamases together with a significant antibacterial activity. The later contribution had prompted us to demonstrate that the 4-methyl-5-oxacephem 6 related to cephalosporin 5 could be obtained starting from L-rhamnal 7..$^{5}$ The formation of the six-membered oxazine ring proceeded via an intramolecular ester condensation in $\mathbf{8}$. 


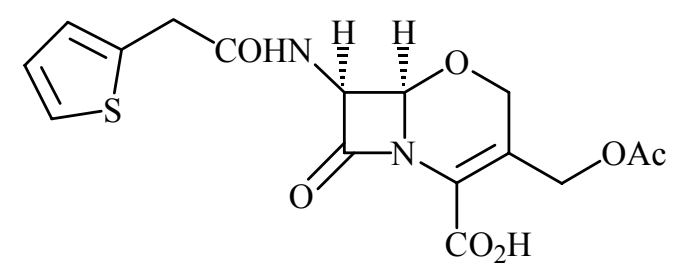

(士) 5-oxacephalotin

1<smiles>[R]C(=O)N[C@]1(O)C(=O)N2C(C(=O)O)=C(CSc3nnnn3[R2])CO[C@H]21</smiles>

3: $\mathrm{R}^{1}=p-\mathrm{HOC}_{6} \mathrm{H}_{4}\left(\mathrm{CO}_{2} \mathrm{H}\right) \mathrm{CH}, \mathrm{R}^{2}=\mathrm{CH}_{3}$ 4: $\mathrm{R}^{1}=\mathrm{CHF}_{2} \mathrm{SCH}_{2}, \mathrm{R}^{2}=\mathrm{HOCH}_{2} \mathrm{CH}_{2}$<smiles>COC(=O)C1=C(OC(C)=O)[C@@H](C)O[C@@H]2[C@@H](COC(C)=O)C(=O)N12</smiles>

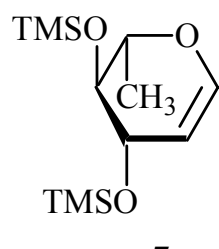

7<smiles>Cn1nnnc1SCC1=C(C(=O)O)N2C(=O)[C@@H](NC(=O)O)[C@H]2OC1</smiles>

(士) 5-oxacephamandol

Very recently we have shown ${ }^{6}$ that our new methodology, that utilizes the 4-vinyloxyazetidin-2-one (9) ${ }^{7}$ as a starting synthon, opens an entry to a range of compounds possessing the 4-methyl-5-oxacepham skeleton, providing that the ethyl lactate is used as a chiral starting material (Scheme 1). ${ }^{6}$<smiles>C=COC1CC(=O)NC1CC(=O)C(C)OCCCC</smiles><smiles>C=COC1CC(=O)N1CC(=C)C(C)[C@H](C)OCCCC</smiles>

Scheme 1 
The present paper describes the introduction of the alkoxycarbonyl function at $\mathrm{C}-2$ of $(4 S, 6 R)$ 3-keto-4-methyl-5-oxacepham 10. The alkoxycarbonylation of the enolate of $\mathbf{1 0}$ should proceed via the intermediate $11(\mathrm{R}=\mathrm{H})$, related to the product of the ester condensation performed on 8 $\left[\mathbf{1 1}\left(\mathrm{R}=\mathrm{CH}_{2} \mathrm{OTBS}\right)\right] .{ }^{5}$ Hence, we were able to expect a positive outcome of the reaction.

\section{Results and Discussion}<smiles>[R][C@]1([2H])C(=O)N2C(C(=O)O[O-])=C([O-])C(C)O[C@H]21</smiles>

11: $\mathrm{R}=\mathrm{H}, \mathrm{CH}_{2} \mathrm{OTBS}$

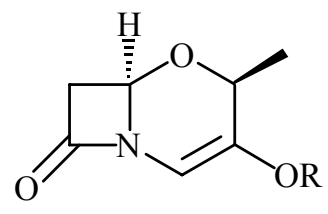

12: $\mathrm{R}=\mathrm{COOEt}$

21: $\mathrm{R}=\mathrm{TMS}$

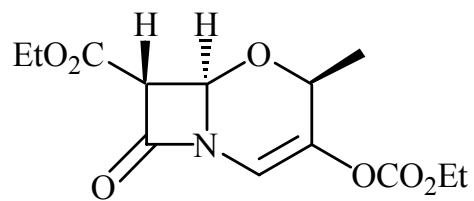

13

A multifunction character of $\mathbf{1 0}$ required generation of the enolate while under the kinetic control of the reaction, which, in turn, anticipated an addition of ketone to the excess of the strong base in order to avoid a self-condensation of the substrate. ${ }^{8}$

Reaction of 10 with 1.1 equiv. of $\mathrm{HMDSLi}$ at $-78^{\circ} \mathrm{C}$ followed by the addition of ethyl chloroformate provided only the product of $O$-acylation (12). The same reaction carried out with 2.5 equiv. of the base led to a mixture of $\mathbf{1 2}$ and $\mathbf{1 3}$.

Softer base, the ethyl cyanoformate and HMDSK gave after acetylation a mixture of three products 14, 15 and 16 in a ratio 1:3.3:1.2, respectively (Scheme 2). Acetylation of a post reaction mixture was necessary since expected $\beta$-ketoesters have been found to be unstable. ${ }^{5}$

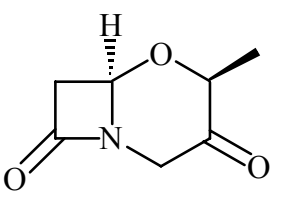

10

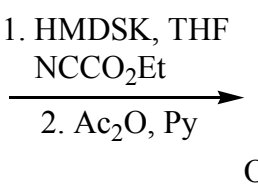

14

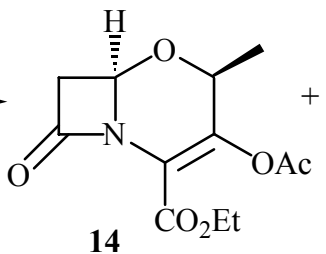

$\mathrm{CO}_{2} \mathrm{Et}$

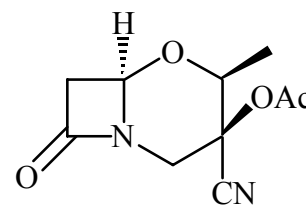

15

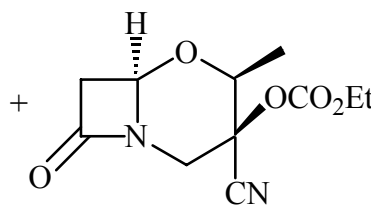

16

Scheme 2

Configuration of $\mathbf{1 5}$ and $\mathbf{1 6}$ was assigned under the assumption of the anti-addition of a cyano group to the 4-methyl substituent of the substrate.

Addition of HMPA to the reaction mixture ${ }^{9}$ resulted, after acetylation, of formation of two products: $14(10 \%)$ and $17(34 \%)$. Configuration of 17 was assigned by analogy to the related compound 22.

It was found that the ketone $\mathbf{1 0}$ undergoes the self-condensation easily to provide, after acetylation, the diacetate $\mathbf{1 8}$ which, upon heating, eliminates elements of acetic acid to give a dimeric compound $\mathbf{1 9}$ in a $50 \%$ overall yield. 


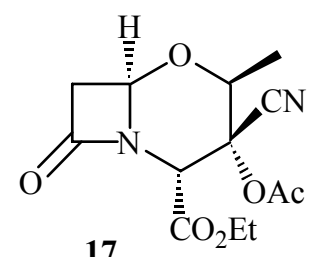

17
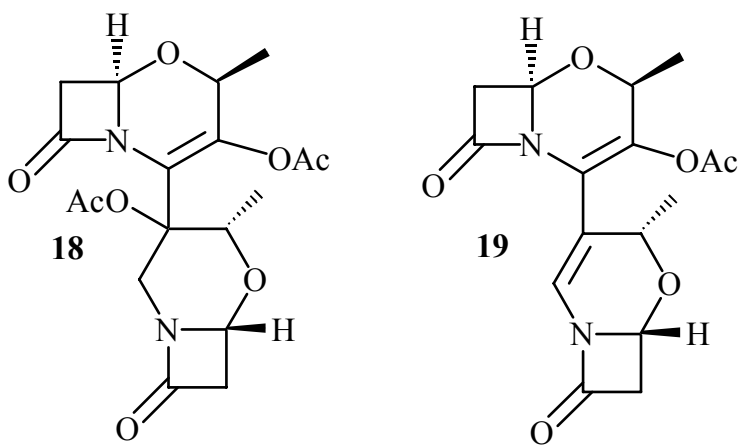

Attempts to introduce the alkoxycarbonyl function by treatment of the enolate of $\mathbf{1 0}$ with ethyl imidazoformate, ${ }^{10}$ diethyl carbonate or the more reactive di- $p$-nitrobenzyl carbonate ${ }^{11}$ were unsuccessful. As the result compound $\mathbf{1 8}$ was obtained as the only isolated product.

An interesting result was noticed when $\mathbf{1 0}$ has been treated with $S$-phenyl $O$-ethyl thiocarbonate ${ }^{12}$ in the presence of HMDSLi and $\mathrm{Bu}_{4} \mathrm{NHSO}_{4}$. The enol carbonate 20, obtained in $11 \%$ yield, was the only isolated product.

It was found that the order of addition of reactants during alkoxycarboylation of the enol of $\mathbf{1 0}$ did not influence significantly the final result of the reaction. The ketone $\mathbf{1 0}$ has three acidic centers at C-2, C-4 and C-7 carbon atoms. Bearing in mind the results of the alkoxycarboylation reactions, one can assume that deprotonation of $\mathbf{1 0}$ leads to a mixture of mono-anions, di-anions and the neutral compound (Scheme 3). The constituents of such a mixture may equilibrate leading, upon reaction with the electrophile, to a variety of alkoxycarbonyl compounds 12-15, 17, 20 and the dimer 18. Moreover the 3-keto group may add cyano group to form corresponding cyanohydrins 15-17. Consequently, complicated mixtures of products are formed.
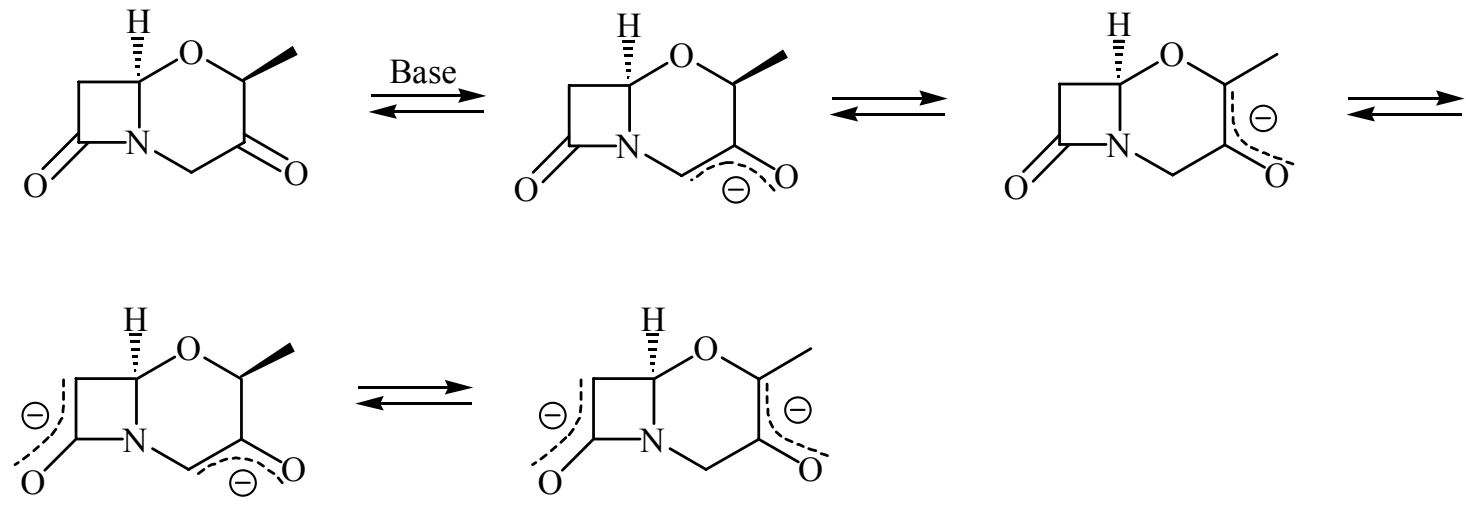

\section{Scheme 3}

Similar results of alkoxycarbonylation were obtained when the silyl vinyl ether 21 (Scheme 4) was subjected to the reaction with ethyl cyanoformate in the presence of tris(dimethylamino)sulfur (trimethylsilyl)difluoride (TASF). Desired products $\mathbf{1 4}$ and $\mathbf{2 2}$ were obtained in low yield only accompanied by undesired nitrile $\mathbf{1 5 .}$ 


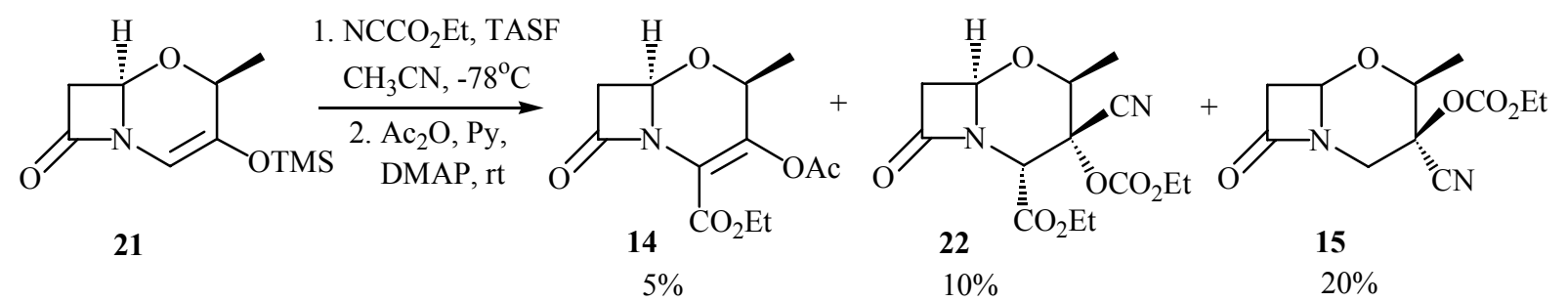

\section{Scheme 4}

The configuration of 22 was proved by analyzing the ${ }^{3} \mathrm{~J}_{\mathrm{CH}}$ coupling constants of the nitrile carbon atom. It has been found by Kingsbury and Wang $^{13}$ that the value of vicinal coupling constants in a six-membered ring depends on the relative location of nitrile substituent and the neighboring protons. For the axial-axial array, the ${ }^{3} \mathrm{~J}_{\mathrm{CH}}$ amounts to $9.2 \pm 0.5 \mathrm{~Hz}$ whereas for the respective axial-equatorial array its value is about $1.7 \mathrm{~Hz}$ (in a conformationally labile systems the value of such a coupling constant may increase to $3.5-4.6 \mathrm{~Hz}) .{ }^{13}$ In the case of compound 22 the ${ }^{3} \mathrm{~J}_{\mathrm{C}, \mathrm{H}-4}=7.8 \mathrm{~Hz}$ whereas the ${ }^{3} \mathrm{~J}_{\mathrm{C}, \mathrm{H}-2}=4.8 \mathrm{~Hz}$. These results suggest the pseudoaxial orientation of the nitrile cis to the methyl and trans to the ethoxycarbonyl group. The relative location of substituents at C-2, -3 and -4 of carbon atoms can be a consequence of the fast equilibration before the final product is quenched by acetylation.

Hydrogenation of the nitrile group in $\mathbf{2 2}$ afforded $\mathbf{2 3}$ which is the result of migration of the acyl from oxygen to the nitrogen atom. The existence of NOE's between both protons of the methylene group at $\mathrm{C}-3$ and vicinal $\mathrm{H}-2$ and $\mathrm{CH}_{3}-4$ supports the assignment made by the analysis of ${ }^{3} \mathrm{~J}_{\mathrm{CH}}$ coupling constants for the nitrile 22.

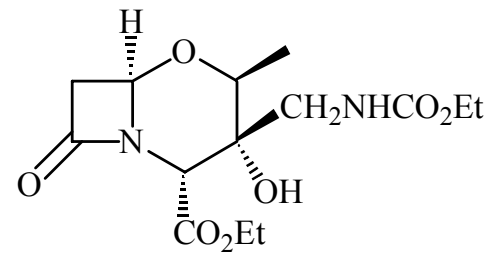

23

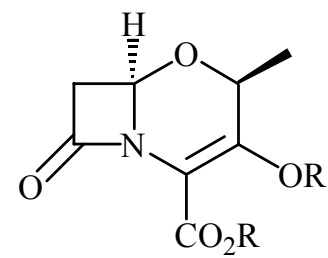

24: $\mathrm{R}=\mathrm{H}$

25: $\mathrm{R}=\mathrm{Me}$

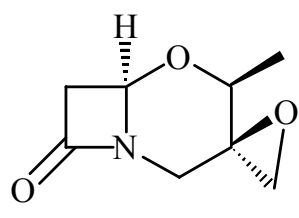

26

Direct carboxylation of the anion $\mathbf{1 0}$ with the carbon dioxide, following closely the known procedure $^{14}$ failed due to the low stability of the acid which underwent fast decarboxylation. As a result, instead of expected methylation of $\mathbf{2 4}$ with diazomethane, which should provide the ester 25, the epoxide $\mathbf{2 6}$ was obtained which likely is a product of addition of diazomethane to the carbonyl group of $\mathbf{1 0}$. The configuration of $\mathbf{2 6}$ was assigned by anticipation of the approach of diazomethane anti to the methyl substituent. Due to the presumed conformational flexibility of the spiroepoxide, the NOE's did not provide an unequivocal assignment.

For comparison we also performed the ethoxycarbonylation of racemic 4,4-dimethyl-5oxacepham $\mathbf{2 7}$ as well as compound $\mathbf{2 8}$ which is the C-6-epimer of $\mathbf{1 0}{ }^{6}$ 
<smiles>CC1(C)OC2CC(=O)N2CC1=O</smiles>

(士) 27<smiles>[CH][C@@H]1O[C@H]2CC(=O)N2CC1=O</smiles>

28<smiles>CCOC(=O)C1=C(OCC)C(C)(C)OC2CC(=O)N12</smiles>

(士) $29 \quad \mathrm{CO}_{2} \mathrm{Et}$<smiles>CCOC(=O)O[C@@]1(C#N)CN2C(=O)C[C@H]2OC1(C)C</smiles>

(士) 30

In the case of $\mathbf{2 7}$ under the same conditions as used for the ethoxycarbonylation of $\mathbf{1 0}$ (THF/HMPA), we obtained 29 (10\%) accompanied by 30 (25\%).

The ethoxycarbonylation of 28, under the same conditions yielded three products 31-33 (Scheme 5). In neither case we did notice the presence of even a trace of products related to 17.
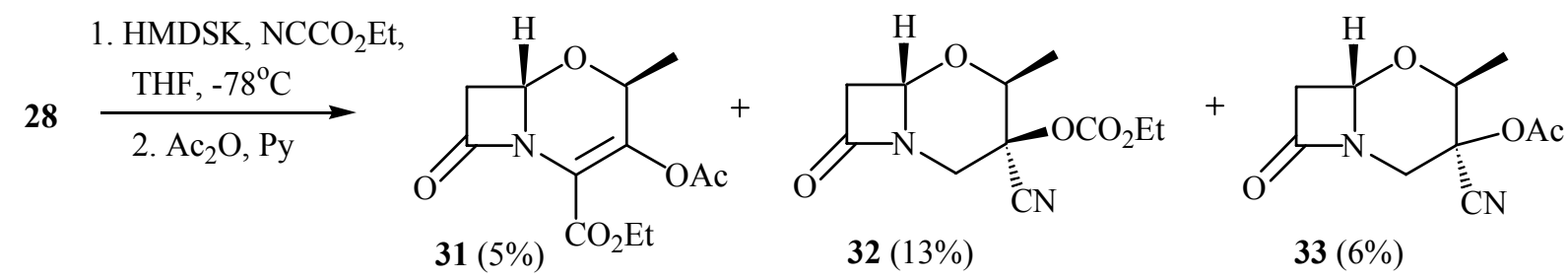

Scheme 5

Configuration of 30, 32 and 33 was assigned assuming the exo-approach to 27 anti to the 4methyl group in $\mathbf{2 8}$, respectively.

\section{Conclusions}

3-Keto-5-oxacephams 10, 27 and 28 have several acidic centers. Deprotonation of these compounds leads to a number of mono- and di-anions which may also equilibrate. In addition, the free keto group may add $\mathrm{CN}^{-}$anion derived from ethyl cyanoformate to form corresponding cyanohydrin or its ester. As a consequence of that, a mixture of products is formed, in which desired compounds 14, 29 and $\mathbf{3 1}$ are obtained in low yield only.

\section{Experimental Section}

General Procedures. Melting points were determined on a Koefler hot-stage apparatus. NMR spectra were recorded using Brucker Avance 500 instrument. IR spectra were recorded on a Perkin-Elmer FTIR Spectrum 200 spectrophotometer. UV spectra were measured on a Cary 100 spectrophotometer in acetonitrile. CD spectra were recorded between 180-400 nm at room temperature with a JASCO J-715 spectropolarimeter using acetonitrile solutions. Mass spectra were recorded using AMD-604 Inectra GmbH and HPLC-MS with Mariner and API 356 
detectors. Optical rotations were measured using a JASCO P 3010 polarimeter at $22 \pm 3{ }^{\circ} \mathrm{C}$. Column chromatography was performed using E. Merck Kiesel Gel (230-400 mesh).

(4S, 6R) 3-Ethoxycarbonyloxy-4-methyl-5-oxa-2-cephem (12). To a solution of 10 (23 mg, $0.15 \mathrm{mmol})$ in THF $(4 \mathrm{~mL})$, cooled to $-78^{\circ} \mathrm{C}$, HMDSLi $(165 \mu \mathrm{L}, 1 \mathrm{M}$ solution in THF, 0.17 mmol) was added dropwise. Subsequently, ethyl chloroformate (16 $\mu \mathrm{L}, 0.17 \mathrm{mmol})$ was added and the mixture was left for $1 \mathrm{~h}$ at $-78^{\circ} \mathrm{C}$. The temperature was allowed to rise to $0^{\circ} \mathrm{C}$ and the mixture was poured into cold water and extracted with $t$-BuOMe $(3 \times 5 \mathrm{~mL})$. The combined extracts were dried over $\mathrm{MgSO}_{4}$ and concentrated. The crude product was purified by chromatography using AcOEt/hexane 20:80 v/v as an eluant to afford of 12 (4 mg, 20\%). Syrup; $[\alpha]_{\mathrm{D}}+4.30\left(\mathrm{c} 1.30, \mathrm{CH}_{2} \mathrm{Cl}_{2}\right)$; IR $\left(\mathrm{CH}_{2} \mathrm{Cl}_{2}\right) 1780,1765 \mathrm{~cm}^{-1} ;{ }^{1} \mathrm{H}$ NMR $\left(\mathrm{CDCl}_{3}\right) 1.34$ (t, 3H, $J .1$ $\left.\mathrm{Hz}, \mathrm{CH}_{2} \mathrm{CH}_{3}\right), 1.34$ (d, 3H, J 6.6 Hz, Me), 2.96 (dd, 1H, J 15.8, $\left.1.3 \mathrm{~Hz}, \mathrm{H}_{\mathrm{a}}-7\right), 3.26$ (br dd, $1 \mathrm{H}, J$ 15.8, $3.8 \mathrm{~Hz}, \mathrm{H}_{\mathrm{b}}-7$ ), 4.26 (q, 2H, J 7.1 Hz, $\mathrm{CH}_{2} \mathrm{CH}_{3}$ ), 4.74 (qd, 1H, J 6.6, $1.5 \mathrm{~Hz}, \mathrm{H}-4$ ), 5.13 (dd, $1 \mathrm{H}, J 3.7,1.2 \mathrm{~Hz}, \mathrm{H}-6), 6.63$ (d, 1H, $J 1.3 \mathrm{~Hz}, \mathrm{H}-2) ;{ }^{13} \mathrm{C} \mathrm{NMR}\left(\mathrm{CDCl}_{3}\right) \delta 14.1,16.8,44.6,65.3$, 70.1, 76.0, 112.3, 140.2, 152.9, 166.0; MS (EI-HR) m/z: M+calcd for $\mathrm{C}_{10} \mathrm{H}_{13} \mathrm{NO}_{5}$ : 227.07937. Found: 227.07986; Anal. Calcd for $\mathrm{C}_{10} \mathrm{H}_{13} \mathrm{NO}_{5}$ (227.22): C, 52.86; H, 5.77; N, 6.16. Found: C, $53.15 ; \mathrm{H}, 6.06 ; \mathrm{N}, 5.92$.

$(4 S, \quad 6 R, \quad 7 R)$ 7-Ethoxycarbonyl-3-ethoxycarbonyloxy-4-methyl-5-oxa-2-cephem (13). Compound 10 (47 mg, $0.3 \mathrm{mmol})$ in THF $(5 \mathrm{~mL})$ cooled to $-78^{\circ} \mathrm{C}$ was treated dropwise with HMDSLi $(750 \mu \mathrm{L}, 1 \mathrm{M}$ solution in THF, $0.75 \mathrm{mmol})$. After $5 \mathrm{~min}$. ethyl chloroformate $(72 \mu \mathrm{L}$, $0.75 \mathrm{mmol}$ ) was added. The mixture was left for $0.5 \mathrm{~h}$ at $-78^{\circ} \mathrm{C}$. Subsequently the temperature was allowed to rise to $0^{\circ} \mathrm{C}$, the mixture was poured into cold water and extracted with ethyl acetate $(3 \times 5 \mathrm{~mL})$. Combine extracts were dried over $\mathrm{MgSO}_{4}$ and concentrated. The crude product was separated by chromatography using AcOEt/hexane 20:80 v/v as an eluant to yield: 13 (15 mg, 10\%) and 12 (14 mg, 21\%).

13. syrup; $[\alpha]_{\mathrm{D}}+98.68\left(\mathrm{c} 0.47, \mathrm{CH}_{2} \mathrm{Cl}_{2}\right)$; IR $\left(\mathrm{CH}_{2} \mathrm{Cl}_{2}\right) 1789,1764,1736 \mathrm{~cm}^{-1} ;{ }^{1} \mathrm{H} \mathrm{NMR}\left(\mathrm{CDCl}_{3}\right)$ : 1.35, $1.31\left(2 \mathrm{t}, 6 \mathrm{H}, J 7.1 \mathrm{~Hz}, 2 \mathrm{CH}_{2} \mathrm{CH}_{3}\right), 1.36$ (d, 3H, J 6.7 Hz, Me), 4.01 (d, 1H, J 1.8 Hz, H-7), 4.27, $4.26\left(2 \mathrm{q}, 4 \mathrm{H}, J 7.1 \mathrm{~Hz}, \mathrm{CH}_{2} \mathrm{CH}_{3}\right.$ ), $4.76(\mathrm{qd}, 1 \mathrm{H}, J 6.7,1.5, \mathrm{H}-4), 5.35$ (d, 1H, J 1.2 Hz, H6), $6.68(\mathrm{~d}, 1 \mathrm{H}, J 1.4 \mathrm{~Hz}, \mathrm{H}-2) .{ }^{13} \mathrm{C} \mathrm{NMR}\left(\mathrm{CDCl}_{3}\right) \delta 14.1,14.1,16.8,61.5,62.1,65.4,70.4$, 77.9, 112.0, 140.8, 152.7, 160.1, 164.9; MS (HR-EI) m/z: $\mathrm{M}^{+}$calcd for $\mathrm{C}_{13} \mathrm{H}_{17} \mathrm{NO}_{7}: 299.10050$. Found: 299.10010; Anal. Calcd for $\mathrm{C}_{13} \mathrm{H}_{17} \mathrm{NO}_{7}$ (299.28): C, 52.17; H, 5.73; N, 4.68. Found: C, $53.82 ; \mathrm{H}, 6.47 ; \mathrm{N}, 4.43$.

$(4 S, 6 R)$ 3-Acetoxy-2-ethoxycarbonyl-4-methyl-5-oxa-2-cephem (14), (3R, 4S, 6R) 3acetoxy-3-cyano-4-methyl-5-oxacepham (15) and $(3 R, \quad 4 S, \quad 6 R)$ 3-cyano-3ethoxycarbonyloxy-4-methyl-5-oxacepham (16). To a solution of 10 (41 $\mathrm{mg}, 0.26 \mathrm{mmol}$ ) in THF (1.5 mL), ethyl cyanoformate $(29 \mu \mathrm{L}, 0.29 \mathrm{mmol})$ was added. The mixture was cooled to $78^{\circ} \mathrm{C}$, treated with HMDSK $(610 \mu \mathrm{L}, 0.5 \mathrm{M}$ solution in toluene, $0.3 \mathrm{mmol})$ and left for $1.5 \mathrm{~h}$ while the temperature was maintained. Subsequently, the reaction was quenched by addition of acetic acid $(22 \mu \mathrm{L})$, pyridine $(1 \mathrm{~mL})$ and acetic anhydride $(0.5 \mathrm{~mL})$. The temperature was allowed to rise to room temperature, the mixture was poured into water and extracted with ethyl 
acetate $(3 \times 2 \mathrm{~mL})$. The combined extracts were dried over $\mathrm{MgSO}_{4}$, concentrated and purified by chromatography using AcOEt/hexane as an eluant to afford compounds 14 (7 mg, 10\%), 15 (7 $\mathrm{mg}, 12 \%)$ and $\mathbf{1 6}(22 \mathrm{mg}, 33 \%)$.

14. syrup; $[\alpha]_{\mathrm{D}}+140.35$ (c $\left.0.17, \mathrm{CH}_{2} \mathrm{Cl}_{2}\right)$; IR $\left(\mathrm{CH}_{2} \mathrm{Cl}_{2}\right) 1791,1725 \mathrm{~cm}^{-1} ;{ }^{1} \mathrm{H}$ NMR $\left(\mathrm{CDCl}_{3}\right): 1.32$ (t, $3 \mathrm{H}, J 7.1 \mathrm{~Hz}, \mathrm{CH}_{2} \mathrm{CH}_{3}$ ), 1.41 (d, 3H, $J 6.7 \mathrm{~Hz}, \mathrm{Me}$ ), 2.24 (s, 3H, Ac), 2.90 (dd, $1 \mathrm{H}, J 15.7,0.8$ $\left.\mathrm{Hz}, \mathrm{H}_{\mathrm{a}}-7\right), 3.34$ (dd, $\left.1 \mathrm{H}, J 15.7,3.8 \mathrm{~Hz}, \mathrm{H}_{\mathrm{b}}-7\right), 4.30,4.25\left(2 \mathrm{q}, 2 \mathrm{H}, J 7.1 \mathrm{~Hz}, \mathrm{CH}_{2} \mathrm{CH}_{3}\right), 4.54$ (q, $1 \mathrm{H}, J 6.7 \mathrm{~Hz}, \mathrm{H}-4), 5.14(\mathrm{dd}, 1 \mathrm{H}, J 3.8,0.7 \mathrm{~Hz}, \mathrm{H}-6) ;{ }^{13} \mathrm{C} \mathrm{NMR}\left(\mathrm{CDCl}_{3}\right) \delta 14.0\left(\mathrm{CH}_{2} \mathrm{CH}_{3}\right), 17.1$ $\left(\mathrm{CH}_{3}\right), 20.7\left(\mathrm{COCH}_{3}\right), 44.8(\mathrm{C}-7), 61.7\left(\mathrm{CH}_{2} \mathrm{CH}_{3}\right), 70.5(\mathrm{C}-4), 76.3(\mathrm{C}-6), 118(\mathrm{C}-2), 146.9$ (C-3), $160.0\left(\mathrm{C}_{2}-\mathbf{C O}\right), 165.8(\mathrm{C}-8), 167,8\left(\mathrm{COCH}_{3}\right)$; MS (HR-LSIMS) $\mathrm{m} / \mathrm{z}:(\mathrm{M}+\mathrm{H})^{+}$calcd for $\mathrm{C}_{12} \mathrm{H}_{16} \mathrm{NO}_{6}: 270.09776$. Found: 270.09871.

15. syrup; IR $\left(\mathrm{CH}_{2} \mathrm{Cl}_{2}\right) 1786 \mathrm{~cm}^{-1} ;{ }^{1} \mathrm{H}$ NMR $\left(\mathrm{CDCl}_{3}\right): 1.49(\mathrm{~d}, 3 \mathrm{H}, J 6.1 \mathrm{~Hz}, \mathrm{Me}), 2.14(\mathrm{~s}, 3 \mathrm{H}$, Ac), 2.97 (dd, 1H, $J$ 13.7, $\left.1.6 \mathrm{~Hz}, \mathrm{H}_{\mathrm{a}}-2\right), 3.03$ (dd, $\left.1 \mathrm{H}, J 15.3,0.7 \mathrm{~Hz}, \mathrm{H}_{\mathrm{a}}-7\right), 3.24$ (ddd, $1 \mathrm{H}, J$ 15.3, 3.4, $\left.1.6 \mathrm{~Hz}, \mathrm{H}_{\mathrm{b}}-7\right), 3.86$ (q, $\left.1 \mathrm{H}, J 6.1 \mathrm{~Hz}, \mathrm{H}-4\right), 5.05$ (d, 1H, $\left.J 13.7 \mathrm{~Hz}, \mathrm{H}_{\mathrm{b}}-2\right), 5.09$ (br d, $1 \mathrm{H}, J 3.1 \mathrm{~Hz}, \mathrm{H}-6) ;{ }^{13} \mathrm{C} \mathrm{NMR}\left(\mathrm{CDCl}_{3}\right) \delta 15.9,20.5,45.6,46.2,69.3,74.8,77.9,114.6,166.5$, 167.8; MS (HR-LSIMS) m/z: $(\mathrm{M}+\mathrm{H})^{+}$calcd for $\mathrm{C}_{10} \mathrm{H}_{13} \mathrm{~N}_{2} \mathrm{O}_{4}$ : 225.08753. Found: 225.08819 .

16. syrup; $[\alpha]_{\mathrm{D}}+54.20\left(\mathrm{c} 0.2, \mathrm{CH}_{2} \mathrm{Cl}_{2}\right)$; IR $\left(\mathrm{CH}_{2} \mathrm{Cl}_{2}\right) 1785,1767 \mathrm{~cm}^{-1} ;{ }^{1} \mathrm{H} \mathrm{NMR}\left(\mathrm{CDCl}_{3}\right): 1.35(\mathrm{t}$, $\left.3 \mathrm{H}, J 7.1 \mathrm{~Hz}, \mathrm{CH}_{2} \mathrm{CH}_{3}\right), 1.51(\mathrm{~d}, 3 \mathrm{H}, J 6.1 \mathrm{~Hz}, \mathrm{Me}), 3.03\left(\mathrm{dd}, 1 \mathrm{H}, J 13.7,1.5 \mathrm{~Hz}, \mathrm{H}_{\mathrm{a}}-2\right), 3.04$ (dd, $1 \mathrm{H}, J 15.4,0.6 \mathrm{~Hz}, \mathrm{H}_{\mathrm{a}}-7$ ), 3.24 (ddd, $1 \mathrm{H}, J 15.4,3.3,1.5 \mathrm{~Hz}, \mathrm{H}_{\mathrm{b}}-7$ ), 3.84 (q, $1 \mathrm{H}, J 6.1 \mathrm{~Hz}$, H-4) 4.29 (2q, 2H, $\left.J 7.1 \mathrm{~Hz}, \mathrm{CH}_{2} \mathrm{CH}_{3}\right), 5.06\left(\mathrm{~d}, 1 \mathrm{H}, J 13.7 \mathrm{~Hz}, \mathrm{H}_{\mathrm{b}}-2\right), 5.08(\mathrm{~d}, 1 \mathrm{H}, J 3.2 \mathrm{~Hz}$, $\mathrm{H}-6) ;{ }^{13} \mathrm{C}$ NMR $\left(\mathrm{CDCl}_{3}\right) \delta 14.0\left(\mathrm{CH}_{2} \mathrm{CH}_{3}\right), 15.9\left(\mathrm{CH}_{3}\right), 45.6(\mathrm{C}-7), 46.2(\mathrm{C}-2), 65.8\left(\mathrm{CH}_{2} \mathrm{CH}_{3}\right)$, $70.8(\mathrm{C}-3), 74.9(\mathrm{C}-4), 77.9(\mathrm{C}-6), 114.5(\mathrm{CN}), 151.6\left(\mathrm{C}_{3}-\mathrm{OCO}\right), 166.4(\mathrm{C}-8) ;{ }^{1} \mathrm{H}$ NMR $\left(\mathrm{C}_{6} \mathrm{D}_{6}\right)$ 0.89 (t, 3H, J 7.1 Hz, $\mathrm{CH}_{2} \mathrm{CH}_{3}$ ), 1.35 (d, 3H, J 6.1 Hz, Me), 2.35 (br d, $\left.1 \mathrm{H}, J 13.6 \mathrm{~Hz}, \mathrm{H}_{\mathrm{a}}-2\right)$, 2.51 (br dd, 1H, $J 15.1,1.8 \mathrm{~Hz}, \mathrm{H}_{\mathrm{a}}-7$ ), 2.64 (d, $\left.1 \mathrm{H}, J 15.1 \mathrm{~Hz}, \mathrm{H}_{\mathrm{b}}-7\right), 3.11$ (q, $1 \mathrm{H}, J 6.1 \mathrm{~Hz}$, $\mathrm{H}-4), 3.82,3,78\left(2 \mathrm{q}, 2 \mathrm{H}, J 7.1 \mathrm{~Hz}, \mathrm{CH}_{2} \mathrm{CH}_{3}\right), 4.13$ (d, $\left.1 \mathrm{H}, J 3.0 \mathrm{~Hz}, \mathrm{H}-6\right), 5.13$ (d, 1H, J 13.6 $\mathrm{Hz}, \mathrm{H}_{\mathrm{b}}-2$ ); MS (HR-ESI) m/z: $(\mathrm{M}+\mathrm{Na})^{+}$calcd for $\mathrm{C}_{11} \mathrm{H}_{14} \mathrm{~N}_{2} \mathrm{O}_{5} \mathrm{Na}$ : 277.0795. Found: 277.0821 . Anal. Calcd for $\mathrm{C}_{11} \mathrm{H}_{14} \mathrm{~N}_{2} \mathrm{O}_{5}$ (254.24): C, 51.97; H, 5.55; N, 11.02. Found: C, 52.00; H, 5.68; N, 10.72 .

(2R, 3S, 4S, 6R) 3-Acetoxy-3-cyano-2-ethoxycarbonyl-4-methyl-5-oxacepham (17). To a solution of HMDSLi $(180 \mu \mathrm{L}, 1 \mathrm{M}$ in THF, $0.18 \mathrm{mmol})$ in THF $(0.3 \mathrm{~mL})$, cooled to $-78^{\circ} \mathrm{C}$, compound $10(25 \mathrm{mg}, 0.16 \mathrm{mmol})$ dissolved in THF $(0.5 \mathrm{~mL})$ was added dropwise. After 30 min., HMPA $(20 \mu \mathrm{L})$ and ethyl cyanoformate $(19 \mathrm{mg}, 19 \mu \mathrm{L}, 0.19 \mathrm{mmol})$ were added and the mixture was kept at $-78^{\circ} \mathrm{C}$ for $2 \mathrm{~h}$. Subsequently, the reaction was quenched by addition of saturated solution of $\mathrm{NH}_{4} \mathrm{Cl}(3 \mathrm{~mL})$ in water and the temperature was allowed to rise to room temperature. The reaction mixture was extracted with $t$-BuOMe $(3 \times 2 \mathrm{~mL})$. The extract was dried and concentrated. The residue was acetylated with $\mathrm{Ac}_{2} \mathrm{O}$ /pyridine mixture $(1 \mathrm{~mL})$ in the presence of a catalytic amount of DMAP. Subsequently, the solvent was evaporated and the residue was dissolved in ethyl ether and the solution was washed with brine, dried and evaporated. The crude product was separated by chromatography using AcOEt/hexane 30:70 v/v as an eluant to afford $\mathbf{1 4}(5 \mathrm{mg}, 10 \%)$ and $\mathbf{1 7}(16 \mathrm{mg}, 34 \%)$. 
17. syrup; $[\alpha]_{\mathrm{D}}-30.9$ (c $\left.0.75, \mathrm{CH}_{2} \mathrm{Cl}_{2}\right)$; IR $\left(\mathrm{CH}_{2} \mathrm{Cl}_{2}\right) 1790,1743 \mathrm{~cm}^{-1} ;{ }^{1} \mathrm{H} \mathrm{NMR}\left(\mathrm{CDCl}_{3}\right) 1.33(\mathrm{t}$, $3 \mathrm{H}, J 7.2 \mathrm{~Hz}, \mathrm{CH}_{2} \mathrm{CH}_{3}$ ), 1.48 (d, 3H, J 6.2 Hz, Me), 2.14 (s, 3H, Ac), 3.08 (dd, 1H, J 15.4, 0.7 $\left.\mathrm{Hz}, \mathrm{H}_{\mathrm{a}}-7\right), 3.28$ (dd, 1H, J 15.4, $\left.3.4 \mathrm{~Hz}, \mathrm{H}_{\mathrm{b}}-7\right), 4.28,4.22\left(2 \mathrm{q}, 4 \mathrm{H}, J 7.2 \mathrm{~Hz}, 2 \mathrm{CH}_{2} \mathrm{CH}_{3}\right), 4.36$ (q, 1H, J 6.2 Hz, H-4), 5.51 (br d, 1H, J $3.2 \mathrm{~Hz}, \mathrm{H}-6$ ), 5.63 (s, 1H, H-2); MS (HR-EI) m/z: M calcd for $\mathrm{C}_{13} \mathrm{H}_{16} \mathrm{~N}_{2} \mathrm{O}_{6}: 296.1002$. Found: 396.1008 .

$\left(4 S, 6 R, 4^{\prime} S, 6^{\prime} R\right)$ 3-Acetoxy-2-[1'-aza-4'-methyl-5'-oxa-bicyclo[4.2.0]oct-2'-en-8'-on-3'-yl]-4methyl-5-oxa-2-cephem (19) and $\left(4 S, 6 R, 3^{\prime} R, 4^{\prime} S, 6^{\prime} R\right)$ 3-acetoxy-2-[3'-acetoxy-1'-aza-4'methyl-5'-oxa-bicyclo[4.2.0]-octan-8'-on-3'-yl]-4-methyl-5-oxa-2-cephem (18). A solution of 10 (17 mg, $0.11 \mathrm{mmol})$ in THF $(1 \mathrm{~mL})$ cooled to $-78^{\circ} \mathrm{C}$ was treated with HMDSK $(260 \mu \mathrm{L}, 0.5$ $\mathrm{M}$ in toluene, $0.13 \mathrm{mmol}$ ). The temperature was then allowed to rise to $0^{\circ} \mathrm{C}$ and the mixture was left for $1 \mathrm{~h}$. Subsequently, pyridine $(100 \mu \mathrm{L}), \mathrm{Ac}_{2} \mathrm{O}(50 \mu \mathrm{L})$ and a catalytic amount of DMAP were added. The mixture was refluxed for $5 \mathrm{~min}$., then poured into cold water and extracted with AcOEt $(3 \times 2 \mathrm{~mL})$. The combined extracts were dried over $\mathrm{MgSO}_{4}$ and concentrated. The crude mixture was separated by chromatography using AcOEt/hexane 20:80 v/v as an eluant to afford $19(20 \mathrm{mg}, 30 \%)$. A minute amount of 18 was isolated, as well.

19. syrup; $[\alpha]_{\mathrm{D}}-57.58\left(\mathrm{c} 0.19, \mathrm{CHCl}_{3}\right)$; IR $\left(\mathrm{CH}_{2} \mathrm{Cl}_{2}\right) 1779,1770 \mathrm{~cm}^{-1} ;{ }^{1} \mathrm{H} \mathrm{NMR}\left(\mathrm{CDCl}_{3}\right): 1.31$ (d, $\left.3 \mathrm{H}, J 6.6 \mathrm{~Hz}, \mathrm{Me}\left(\mathrm{Me}^{\prime}\right)\right), 1.36$ (d, 3H, J 6.7 Hz, Me' (Me)), 2.15 (s, 3H, Ac), 2.93 (dd, 1H, $J$ 15.8, $\left.1.2 \mathrm{~Hz}, \mathrm{H}_{\mathrm{a}}-7\left(7^{\prime}\right)\right), 3.03\left(\mathrm{dd}, 1 \mathrm{H}, J 16.0,1.4 \mathrm{~Hz}, \mathrm{H}_{\mathrm{a}}-7^{\prime}(7)\right), 3.24$ (dd, $1 \mathrm{H}, J 15.8,3.8 \mathrm{~Hz}$, $\left.\mathrm{H}_{\mathrm{b}}-7\left(7^{\prime}\right)\right), 3.28\left(\mathrm{dd}, 1 \mathrm{H}, J 16.0,3.8 \mathrm{~Hz}, \mathrm{H}_{\mathrm{b}}-7^{\prime}(7)\right), 4.71\left(\mathrm{q}, 1 \mathrm{H}, J 6.6 \mathrm{~Hz}, \mathrm{H}-4\left(4^{\prime}\right)\right), 4.81$ (qd, 1H, $J$ 6.7, $\left.1.6 \mathrm{~Hz}, \mathrm{H}-4^{\prime}(4)\right), 5.10$ (dd, 1H, J 3.8, $1.2 \mathrm{~Hz}, \mathrm{H}-6\left(6^{\prime}\right), 5.16$ (dd, 1H, J 3.8, $1.1 \mathrm{~Hz}$, $\mathrm{H}-6{ }^{\prime}(6), 6.56\left(\mathrm{~d}, 1 \mathrm{H}, J 1.4 \mathrm{~Hz}, \mathrm{H}-2^{\prime}\right) ;{ }^{13} \mathrm{C} \mathrm{NMR}\left(\mathrm{CDCl}_{3}\right) \delta 17.4,18.3,20.4,44.4(\mathrm{t}), 45.4(\mathrm{t})$, 70.5, 71.6, 76.1, 76.3, 96.1 (s), 116.2 (s), 122.2, 137.7 (s), 165.3 (s), 165.5 (s), 169.4 (s); MS (HR-EI) m/z: (M) ${ }^{+}$calcd for $\mathrm{C}_{16} \mathrm{H}_{18} \mathrm{~N}_{2} \mathrm{O}_{6}$ : 334.11649. Found: 334.11626 .

18. syrup; IR $\left(\mathrm{CH}_{2} \mathrm{Cl}_{2}\right) 1772 \mathrm{~cm}^{-1} ;{ }^{1} \mathrm{H} \mathrm{NMR}\left(\mathrm{CDCl}_{3}\right): 1.25$ (d, 3H, J 6.6 Hz, Me $\left.\left(\mathrm{Me}^{\prime}\right)\right), 1.33$ (d, $\left.3 \mathrm{H}, J 6.5 \mathrm{~Hz}, \mathrm{Me}^{\prime}(\mathrm{Me})\right), 2.05$ (s, 3H, Ac'), 2.16 (s, 3H, Ac) 2.83 (d, 1H, J $14.9 \mathrm{~Hz}, \mathrm{H}_{\mathrm{a}}-7\left(7^{\prime}\right)$ ), $2.84\left(\mathrm{dd}, 1 \mathrm{H}, J\right.$ 15.8, $\left.0.9 \mathrm{~Hz}, \mathrm{H}_{\mathrm{a}}-7^{\prime}(7)\right), 3.20$ (ddd, 1H, J 14.9, 3.2, $1.2 \mathrm{~Hz}, \mathrm{H}_{\mathrm{b}}-7\left(7^{\prime}\right), 3.28$ (dd, $\left.1 \mathrm{H}, J 15.8,3.7 \mathrm{~Hz}, \mathrm{H}_{\mathrm{b}}-7^{\prime}(7)\right), 3.63$ (dd, 1H, J 14.7, $\left.1.1 \mathrm{~Hz}, \mathrm{H}_{\mathrm{a}}-2^{\prime}\right), 4.40$ (bq, 1H, J $6.5 \mathrm{~Hz}$, $\left.\mathrm{H}-4^{\prime}(4)\right), 4.75$ (d, 1H, J $\left.14.7 \mathrm{~Hz}, \mathrm{H}_{\mathrm{b}}-2^{\prime}\right), 4.94$ (q, 1H, J 6.6 Hz, H-4(4')), 5.12 (d, 1H, J 3.2 Hz, $\mathrm{H}-6\left(6^{\prime}\right), 5.20$ (d, 1H, J 3.6 Hz, H-6'(6); MS (HR-EI) m/z: M calcd for $\mathrm{C}_{18} \mathrm{H}_{22} \mathrm{~N}_{2} \mathrm{O}_{8}$ : 394.1376. Found: 394.1386.

(6R) 3-Ethoxycarbonyloxy-4-methyl-5-oxa-3-cephem (20). To a solution of 10 (68 $\mathrm{mg}, 0.44$ $\mathrm{mmol})$ in THF $(8 \mathrm{~mL})$ pulverized $\mathrm{Bu}_{4} \mathrm{NHSO}_{4}(165 \mathrm{mg}, 0.48 \mathrm{mmol})$ was added. The mixture was cooled to $-78^{\circ} \mathrm{C}$ and treated with HMDSLi ( $1 \mathrm{~mL}, 1 \mathrm{M}$ in THF, $\left.1.0 \mathrm{mmol}\right)$. Subsequently, the temperature was allowed to rise to $-40^{\circ} \mathrm{C}$. After $20 \mathrm{~min}$, the mixture was again cooled to $-78^{\circ} \mathrm{C}$ and treated with $S$-phenyl $O$-ethyl thiocarbonate $(77 \mu \mathrm{L}, 0.48 \mathrm{mmol})$. The temperature was maintained for $20 \mathrm{~min}$. and then it was allowed to rise to $0^{\circ} \mathrm{C}$. The mixture was poured into cold water and extracted with $t$-BuOMe $(3 \times 5 \mathrm{~mL})$. The combined extracts were dried over $\mathrm{MgSO}_{4}$ and concentrated. The crude product was separated by chromatography using AcOEt/hexane 20:80 v/v as an eluant to afford $20(11 \mathrm{mg}, 11 \%) ; \mathrm{mp} 68-69^{\circ} \mathrm{C} ;[\alpha]_{\mathrm{D}}+122.70\left(\mathrm{c} 0.22, \mathrm{CH}_{2} \mathrm{Cl}_{2}\right)$; IR $\left(\mathrm{CH}_{2} \mathrm{Cl}_{2}\right) 1776,1773 \mathrm{~cm}^{-1} ;{ }^{1} \mathrm{H}$ NMR $\left(\mathrm{CDCl}_{3}\right) 1.35\left(\mathrm{t}, 3 \mathrm{H}, J 7.1 \mathrm{~Hz}, \mathrm{CH}_{2} \mathrm{CH}_{3}\right), 1.80(\mathrm{t}, 3 \mathrm{H}, J$ 
$2.0 \mathrm{~Hz}, \mathrm{Me}), 2.90$ (d, 1H, J 15.0 Hz, Ha -7 ), 3.26 (ddd, 1H, J 15.0, 3.1, $1.8 \mathrm{~Hz}, \mathrm{H}_{\mathrm{b}}-7$ ), 3.61 (dm, $\left.1 \mathrm{H}, J 15.8 \mathrm{~Hz}, \mathrm{H}_{\mathrm{a}}-2\right), 4.18\left(\mathrm{dm}, 1 \mathrm{H}, J 15.8 \mathrm{~Hz}, \mathrm{H}_{\mathrm{b}}-2\right), 4.26$ (q, 2H, $\left.J 7.1 \mathrm{~Hz}, \mathrm{CH}_{2} \mathrm{CH}_{3}\right), 5.18$ (d, $1 \mathrm{H}, J 3.1 \mathrm{~Hz}, \mathrm{H}-6) ;{ }^{13} \mathrm{C} \mathrm{NMR}\left(\mathrm{CDCl}_{3}\right) \delta 14.2,14.4,37.8,45.9,65.2,75.0,123.9,144.0,153.4$, 166.9; MS (EI-HR) m/z: $\mathrm{M}^{+}$calcd for $\mathrm{C}_{10} \mathrm{H}_{13} \mathrm{NO}_{5}$ : 227.07937. Found: 227.07992.

(4S, 6R) 3-Trimethylsiloxy-4-methyl-5-oxa-2-cephem (21). A solution of 10 (46 mg, 0.3 $\mathrm{mmol})$ in THF $(2.5 \mathrm{~mL})$, cooled to $-78^{\circ} \mathrm{C}$ was treated successively with LDA $(160 \mu \mathrm{L} 2 \mathrm{M}$ in $\mathrm{THF} /$ heptane/ethylbenzene, $0.33 \mathrm{mmol}$ ) and $\mathrm{TMSCl}(55 \mathrm{mg}, 64 \mu \mathrm{L}, 0.5 \mathrm{mmol})$. The mixture was kept for $5 \mathrm{~min}$ at $-78^{\circ} \mathrm{C}$ and for $1 \mathrm{~h}$ at room temperature. The mixture was then poured into sat. aq. sodium hydrogencarbonate and extracted with hexane $(3 \times 3 \mathrm{~mL})$. The extracts were dried over $\mathrm{MgSO}_{4}$ and concentrated to afford 21 (47 mg, $70 \%$ ): syrup; $[\alpha]_{\mathrm{D}}-28.80$ (c 0.45, $\mathrm{CH}_{2} \mathrm{Cl}_{2}$ ); IR $\left(\mathrm{CH}_{2} \mathrm{Cl}_{2}\right) 1771 \mathrm{~cm}^{-1}$; ${ }^{1} \mathrm{H}$ NMR $\left(\mathrm{CDCl}_{3}\right): 0.22$ (s, 9H, $\left.\mathrm{SiMe}_{3}\right), 1.34(\mathrm{~d}, 3 \mathrm{H}, J 6.6 \mathrm{~Hz}, \mathrm{Me}), 2.86$ (dd, 1H, J 15.4, 1.1 Hz, Ha -7 ), 3.18 (ddd, 1H, $J 15.4,3.5,0.5$ Hz, Hb -7 ), 4.37 (qd, 1H, J 6.6, 1.2 $\mathrm{Hz}, \mathrm{H}-4), 4.98$ (dd, 1H, J 3.5, $0.9 \mathrm{~Hz}, \mathrm{H}-6), 6.01$ (bs, $1 \mathrm{H}, \mathrm{H}-2) .{ }^{13} \mathrm{C} \mathrm{NMR}\left(\mathrm{CDCl}_{3}\right) \delta 0.0,17.5$, 44.2, 71.8, 75.4, 102.8, 144.6, 166.0; MS (HR-EI) m/z: $\mathrm{M}^{+}$calcd for $\mathrm{C}_{10} \mathrm{H}_{17} \mathrm{NO}_{3} \mathrm{Si}: 227.09777$. Found: 227.09761.

(2R, 3S, 4S, 6R) 3-Cyano-2-ethoxycarbonyl-3-ethoxycarbonyloxy-4-methyl-5-oxacepham (22). A solution of silyl ether 21 (36 mg, $0.16 \mathrm{mmol})$ in THF (1 mL) and $\mathrm{CH}_{3} \mathrm{CN}(1 \mathrm{~mL})$ cooled to $-78^{\circ} \mathrm{C}$ was treated with ethyl cyanoformate $(17 \mathrm{mg}, 18 \mu \mathrm{L}, 0.17 \mathrm{mmol}$ ) and TASF (44 $\mathrm{mg}$, $0.16 \mathrm{mmol})$. The mixture was kept at $-78^{\circ} \mathrm{C}$ for $2.5 \mathrm{~h}$. Subsequently, $\mathrm{Ac}_{2} \mathrm{O}(0.3 \mathrm{~mL})$, pyridyne $(0.6 \mathrm{~mL})$ and catalytic amount of DMAP were added. The temperature was then allowed to rise to $20^{\circ} \mathrm{C}$ and the mixture was left for $1 \mathrm{~h}$. The reaction mixture was poured into cold water and extracted with $t$-BuOMe $(3 \times 3 \mathrm{~mL})$. The combined extracts were dried over $\mathrm{MgSO}_{4}$ and concentrated. The crude product was separated by chromatography using $t$-BuOMe/hexane $50: 50 \mathrm{v} / \mathrm{v}$ as an eluant to afford $22(5 \mathrm{mg}, 10 \%), 14(2 \mathrm{mg}, 5 \%)$ and 15 (8 $\mathrm{mg}, 20 \%)$.

22. syrup; IR $\left(\mathrm{CH}_{2} \mathrm{Cl}_{2}\right)$ 1789, $1745 \mathrm{~cm}^{-1} ;{ }^{1} \mathrm{H} \mathrm{NMR}\left(\mathrm{CDCl}_{3}\right): 1.37,1.28(2 \mathrm{t}, 6 \mathrm{H}, J 7.2 \mathrm{~Hz}, 2$ $\mathrm{CH}_{2} \mathrm{CH}_{3}$ ), 1.48 (d, 3H, J $\left.6.2 \mathrm{~Hz}, \mathrm{Me}\right), 3.05$ (dd, 1H, J 15.4, 0.7 Hz, Ha $\left.3.4 \mathrm{~Hz}, \mathrm{H}_{\mathrm{b}}-7\right), 4.32,4.23$ (2q, 4H, J 7.2 Hz, $2 \mathrm{CH}_{2} \mathrm{CH}_{3}$ ), 4.28 (q, 1H, J $\left.6.2 \mathrm{~Hz}, \mathrm{H}-4\right), 5.50$ (bd, $1 \mathrm{H}, J 2.8 \mathrm{~Hz}, \mathrm{H}-6), 5.59(\mathrm{~s}, 1 \mathrm{H}, \mathrm{H}-2) ;{ }^{13} \mathrm{C} \mathrm{NMR}\left(\mathrm{CDCl}_{3}\right) \delta 13.8\left(\mathrm{CH}_{3}\right), 14.0\left(\mathrm{CH}_{3}\right), 15.9\left(\mathrm{CH}_{3}\right)$, $45.7(\mathrm{C}-7), 54.7,62.8\left(\mathrm{CH}_{2} \mathrm{CH}_{3}\right), 66.1\left(\mathrm{CH}_{2} \mathrm{CH}_{3}\right), 71.2,71.4(\mathrm{C}-3), 77.4(\mathrm{C}-6), 113.8(\mathrm{CN})$, $151.4\left(\mathrm{C}_{3}-\mathrm{CO}\right), 165.4\left(\mathrm{C}_{2}-\mathrm{CO}\right), 165.7(\mathrm{C}-8) ; \mathrm{MS}(\mathrm{HR}-\mathrm{ESI}) \mathrm{m} / \mathrm{z}:(\mathrm{M}+\mathrm{Na})^{+}$calcd for $\mathrm{C}_{14} \mathrm{H}_{18} \mathrm{~N}_{2} \mathrm{O}_{7} \mathrm{Na}: 349.1006$. Found: 349.1015.

(2R, 3R, 4S, 6R) 3-Hydroxy-2-ethoxycarbonyl-3-(ethoxycarbonylaminomethyl)-4-methyl-5oxacepham (23). To a solution of $22(5 \mathrm{mg}, 0.016 \mathrm{mmol})$ in $\mathrm{MeOH}(0.5 \mathrm{~mL})$ and AcOEt $(0.5$ $\mathrm{mL})$ was added $\mathrm{Pd}(\mathrm{OH})_{2} / \mathrm{C}(3 \mathrm{mg})$. Upon stirring the mixture was kept under hydrogen atmosphere overnight. Subsequently the solution was filtered through Celite and evaporated. The mixture was evaporated and purified by chromatography using AcOEt/hexane 50:50 v/v as an eluant to afford 23 (3 mg, 60\%): syrup; $[\alpha]_{\mathrm{D}}+29.4\left(\mathrm{c} 0.1, \mathrm{CH}_{2} \mathrm{Cl}_{2}\right) ;{ }^{1} \mathrm{H} \mathrm{NMR}\left(\mathrm{CDCl}_{3}\right)$ : 1.30 (d, $3 \mathrm{H}, J 6.6 \mathrm{~Hz}, \mathrm{Me}), 1.31\left(2 \mathrm{t}, 6 \mathrm{H}, J 7.1 \mathrm{~Hz}, 2 \mathrm{CH}_{2} \mathrm{CH}_{3}\right), 2.78\left(\mathrm{~d}, 1 \mathrm{H}, J 15.1 \mathrm{~Hz}, \mathrm{H}_{\mathrm{a}}-7\right), 3.22$ (dd, $\left.1 \mathrm{H}, J 15.1,3.3 \mathrm{~Hz}, \mathrm{H}_{\mathrm{b}}-7\right), 3.28$ (dd, $\left.1 \mathrm{H}, J 15.1,6.7 \mathrm{~Hz}, \mathrm{H}_{\mathrm{a}}-3^{\prime}\right), 3.65$ (dd, $1 \mathrm{H}, J 15.1,4.6 \mathrm{~Hz}$, $\left.\mathrm{H}_{\mathrm{b}}-3^{\prime}\right), 4.22$ (4q, 4H, J 7.1 Hz, $2 \mathrm{CH}_{2} \mathrm{CH}_{3}$ ), 4.34 (q, 1H, J 6.4 Hz, H-4), 4.78 (s, 1H, H-2), 5.30 
(br s, 1H, OH), $5.32(\mathrm{~s}, 1 \mathrm{H}, \mathrm{NH}), 5.46(\mathrm{~d}, 1 \mathrm{H}, J 3.3 \mathrm{~Hz}, \mathrm{H}-6) ;{ }^{1} \mathrm{H}$ NMR $\left(\mathrm{C}_{6} \mathrm{D}_{6}\right)$ 0.90, $0.91(2 \mathrm{t}$, $\left.6 \mathrm{H}, J 7.1 \mathrm{~Hz}, 2 \mathrm{CH}_{2} \mathrm{CH}_{3}\right) 1.18(\mathrm{~d}, 3 \mathrm{H}, J 6.7 \mathrm{~Hz}, \mathrm{Me}), 2.37$ (d, 1H, J $\left.14.9 \mathrm{~Hz}, \mathrm{H}_{\mathrm{a}}-7\right), 2.51$ (dd, $1 \mathrm{H}, J$ 14.9, $3.3 \mathrm{~Hz}, \mathrm{H}_{\mathrm{b}}-7$ ), 2.94 (dd, 1H, J 15.2, $6.9 \mathrm{~Hz}, \mathrm{H}_{\mathrm{a}}-3^{\prime}$ ), 3.38 (dd, 1H, J 15.3, 4.6 Hz, $\left.\mathrm{H}_{\mathrm{b}}-3^{\prime}\right), 3.86,3.80\left(2 \mathrm{q}, 2 \mathrm{H}, J 7.1 \mathrm{~Hz}, \mathrm{CH}_{2} \mathrm{CH}_{3}\right), 3.98,3.95\left(2 \mathrm{q}, 2 \mathrm{H}, J 7.1 \mathrm{~Hz}, \mathrm{CH}_{2} \mathrm{CH}_{3}\right), 4.57$ (q, $1 \mathrm{H}, J 6.7 \mathrm{~Hz}, \mathrm{H}-4), 5.01$ (s, 1H, H-2), 5.03 (br s, 1H, NH), 5.29 (d, 1H, J $3.3 \mathrm{~Hz}, \mathrm{H}-6), 5.92$ (s, $1 \mathrm{H}, \mathrm{OH})$; MS (HR-ESI) m/z: $(\mathrm{M}+\mathrm{Na})^{+}$calcd for $\mathrm{C}_{14} \mathrm{H}_{22} \mathrm{~N}_{2} \mathrm{O}_{7} \mathrm{Na}: 353.1319$. Found: 353.1322 .

(3R, 4S, 6R) 4-Methyl-3-spirooxirano-5-oxacepham (26). A solution of HMDSLi (340 $\mu \mathrm{L}, 1$ $\mathrm{M}$ in THF $)$ in THF $(0.5 \mathrm{~mL})$ was cooled to $-78^{\circ} \mathrm{C}$ and treated with $\mathbf{1 0}(25 \mathrm{mg}, 0.16 \mathrm{mmol})$. After $10 \mathrm{~min}$, the mixture was cooled to $-180^{\circ} \mathrm{C}$ (liquid nitrogen) and anhydrous carbon dioxide was passed through the solution for $5 \mathrm{~min}$. Subsequently, the temperature was allowed to rise to $60^{\circ} \mathrm{C}$ and the mixture was left for $2 \mathrm{~h}$. The temperature was increased to $-20^{\circ} \mathrm{C}$ and $\mathrm{AcOH}(40$ $\mathrm{mg}, 0.64 \mathrm{mmol}$ ) was added. After $10 \mathrm{~min}$ the mixture was treated with etheral solution of diazomethane. Subsequently, the mixture was poured into water and extracted with AcOEt (3x). The combined extracts were dried and concentrated. The crude product was purified by chromatography using AcOEt/hexane 30:70 v/v as an eluant to afford 26 (11 mg, 41\%): syrup; $[\alpha]_{\mathrm{D}}+15.3\left(\mathrm{c} 0.06, \mathrm{CH}_{2} \mathrm{Cl}_{2}\right.$ ); IR (film z $\left.\mathrm{CHCl}_{3}\right) 1772 \mathrm{~cm}^{-1}$; ${ }^{1} \mathrm{H} \mathrm{NMR}\left(\mathrm{CDCl}_{3}\right)$ : 1.09 (d, 3H, $J 6.4$ $\mathrm{Hz}, \mathrm{Me}$ ), 2.74 (d, 1H, J 4.3 Hz, $\left.\mathrm{H}_{\mathrm{b}}-3^{\prime}\right), 2.85$ (d, 1H, J 15.1 Hz, $\mathrm{H}_{\mathrm{b}}-7$ ), 3.00 (dd, 1H, J 4.3, 1.7 $\mathrm{Hz}, \mathrm{H}_{\mathrm{a}}-3^{\prime}$ ), 3.20 (ddd, $1 \mathrm{H}, J 15.1,3.3,1.7 \mathrm{~Hz}, \mathrm{H}_{\mathrm{a}}-7$ ), 3.32 (dd, $1 \mathrm{H}, J 13.7,1.7 \mathrm{~Hz}, \mathrm{H}_{\mathrm{b}}-2$ ), 3.58 (d, 1H, J $\left.13.7 \mathrm{~Hz}, \mathrm{H}_{\mathrm{a}}-2\right)$, 4.09 (q, 1H, J 6.4 Hz, H-4), 5.14 (d, 1H, J 3.2 Hz, H-6); MS (HR-EI) $\mathrm{m} / \mathrm{z}: \mathrm{M}^{+}$calcd for $\mathrm{C}_{8} \mathrm{H}_{11} \mathrm{NO}_{3}: 169.0739$. Found: 169.0731 .

( \pm ) 3-Acetoxy-2-ethoxycarbonyl-4,4-dimethyl-5-oxa-2-cephem (29) and ( \pm ) 3-cyano-3ethoxycarbonyloxy-4,4-dimethyl-5-oxacepham (30). Compounds 29 and 30 were obtained from 27 (17 mg, $0.1 \mathrm{mmol})$ in THF/HMPA with HMDSK $(240 \mu \mathrm{L}, 0.5 \mathrm{M}$ in toluene, $0.12 \mathrm{mmol})$ and ethyl cyanoformate $(11 \mathrm{mg}, 11 \mu \mathrm{L}, 0.11 \mathrm{mmol})$ at $-78^{\circ} \mathrm{C}$ according to the procedure described for 10 to afford 29 (3 mg, 10\%) and $\mathbf{3 0}(7 \mathrm{mg}, 25 \%)$.

29. syrup; IR (film z CH $\left.\mathrm{Cl}_{2}\right)$ 1784, 1723, $1636 \mathrm{~cm}^{-1}$; ${ }^{1} \mathrm{H} \mathrm{NMR}\left(\mathrm{CDCl}_{3}\right): 1.31$ (t, $3 \mathrm{H}, J 7.1 \mathrm{~Hz}$, $\mathrm{CH}_{2} \mathrm{CH}_{3}$ ), 1.41, 1.44 (2s, 6H, 2Me), 2.24 (s, 3H, Ac), 2.87 (dd, 1H, J 15.6, $0.9 \mathrm{~Hz}, \mathrm{H}_{\mathrm{a}}-7$ ), 3.35 (dd, 1H, J 15.6, 3.7 Hz, $\mathrm{H}_{\mathrm{b}}-7$ ), 4.24, 4.29 (2q, 2H, J 7.1 Hz, $\left.\mathrm{CH}_{2} \mathrm{CH}_{3}\right), 5.21$ (dd, 1H, J 3.7, 0.9 $\mathrm{Hz}, \mathrm{H}-6) ;{ }^{13} \mathrm{C} \mathrm{NMR}\left(\mathrm{CDCl}_{3}\right) \delta 14.0\left(\mathrm{CH}_{2} \mathrm{CH}_{3}\right), 20.9\left(\mathrm{COCH}_{3}\right), 24.6\left(\mathrm{CH}_{3}\right), 25.7\left(\mathrm{CH}_{3}\right), 44.8$ $(\mathrm{C}-7), 61.7\left(\mathrm{CH}_{2} \mathrm{CH}_{3}\right), 72.3(\mathrm{C}-6), 75.7(\mathrm{C}-4), 116.9(\mathrm{C}-2), 149.2(\mathrm{C}-3), 166.0(\mathrm{C}-8), 167.7$ $\left(\mathrm{COCH}_{3}\right), 173.2\left(\mathrm{C}_{2}-\mathrm{CO}\right)$; MS (HR-EI) m/z: $\mathrm{M}^{+}$calcd forC ${ }_{13} \mathrm{H}_{17} \mathrm{NO}_{6}$ : 283.1056. Found: 283.1035 .

30. syrup; IR (film z CH $\left.\mathrm{Cl}_{2}\right) 1781 \mathrm{~cm}^{-1} ;{ }^{1} \mathrm{H} \mathrm{NMR}\left(\mathrm{CDCl}_{3}\right)$ : 1.34 (t, $3 \mathrm{H}, J 7.1 \mathrm{~Hz}, \mathrm{CH}_{2} \mathrm{CH}_{3}$ ), 1.50, $1.56(2 \mathrm{~s}, 6 \mathrm{H}, 2 \mathrm{Me}), 3.04\left(\mathrm{dd}, 1 \mathrm{H}, J 15.3,0.6 \mathrm{~Hz}, \mathrm{H}_{\mathrm{a}}-7\right), 3.16(\mathrm{dd}, 1 \mathrm{H}, J 13.8,1.3 \mathrm{~Hz}$, $\mathrm{H}_{\mathrm{a}}-2$ ), 3.23 (ddd, $\left.1 \mathrm{H}, J 15.3,3.3,1.5 \mathrm{~Hz}, \mathrm{H}_{\mathrm{b}}-7\right), 4.27,4.28\left(2 \mathrm{q}, 2 \mathrm{H}, J 7.1 \mathrm{~Hz}, \mathrm{CH}_{2} \mathrm{CH}_{3}\right), 5.00$ (d, $\left.1 \mathrm{H}, J 13.8 \mathrm{~Hz}, \mathrm{H}_{\mathrm{b}}-2\right), 5.22(\mathrm{~d}, 1 \mathrm{H}, J 3.1 \mathrm{~Hz}, \mathrm{H}-6) ;{ }^{13} \mathrm{C} \mathrm{NMR}\left(\mathrm{CDCl}_{3}\right) \delta 14.0\left(\mathrm{CH}_{2} \mathrm{CH}_{3}\right), 17.6$ $\left(\mathrm{CH}_{3}\right), 25.3\left(\mathrm{CH}_{3}\right), 26.9(\mathrm{C}-2), 42.9\left(\mathrm{CH}_{2} \mathrm{CH}_{3}\right), 45.8(\mathrm{C}-7), 65.7(\mathrm{C}-3), 73.4(\mathrm{C}-6), 76.9(\mathrm{C}-4)$, $115.8(\mathrm{CN}), 151.8\left(\mathrm{C}_{3}-\mathrm{OCO}\right), 167.4(\mathrm{C}-8)$; $\mathrm{MS}(\mathrm{HR}-\mathrm{EI}) \mathrm{m} / \mathrm{z}: \mathrm{M}^{+}$calcd for $\mathrm{C}_{12} \mathrm{H}_{16} \mathrm{~N}_{2} \mathrm{O}_{5}$ : 268.1059. Found: 268.1065. 
(4S, 6S) 3-Acetoxy-2-ethoxycarbonyl-4-methyl-5-oxa-2-cephem (31), (4S, 6S) 3-cyano-3ethoxycarbonyloxy-4-methyl-5-oxa-cepham (32) and (4S, 6S) 3-acetoxy-3-cyano-4-methyl-5oxacepham (33). Compounds 31, 32 and 33 were obtained from 28 according to the procedure described above. The following products were isolated: $31(5 \%), 32(13 \%)$ and $33(6 \%)$.

31. syrup; $[\alpha]_{\mathrm{D}}-7.66\left(\mathrm{c} 0.18, \mathrm{CH}_{2} \mathrm{Cl}_{2}\right)$; IR $\left(\mathrm{CH}_{2} \mathrm{Cl}_{2}\right)$ 1792, 1781, $1725 \mathrm{~cm}^{-1} ;{ }^{1} \mathrm{H} \mathrm{NMR}\left(\mathrm{CDCl}_{3}\right)$ : 1.32 (t, 3H, J 7.2 Hz, $\left.\mathrm{CH}_{2} \mathrm{CH}_{3}\right), 1.41$ (d, 3H, J 6.9 Hz, Me), 2.23 (s, 3H, $\left.\mathrm{COCH}_{3}\right), 2.88(\mathrm{dd}, 1 \mathrm{H}, J$ 15.7, $\left.0.8 \mathrm{~Hz}, \mathrm{H}_{\mathrm{a}}-7\right), 3.36$ (dd, $\left.1 \mathrm{H}, J 15.7,3.5 \mathrm{~Hz}, \mathrm{H}_{\mathrm{b}}-7\right), 4.31,4.26\left(2 \mathrm{q}, 2 \mathrm{H}, J 7.2 \mathrm{~Hz}, \mathrm{CH}_{2} \mathrm{CH}_{3}\right.$ ), 4.56 (q, 1H, J $6.9 \mathrm{~Hz}, \mathrm{H}-4), 5.20(\mathrm{~d}, 1 \mathrm{H}, J 3.6 \mathrm{~Hz}, \mathrm{H}-6) ;{ }^{13} \mathrm{C} \mathrm{NMR}\left(\mathrm{CDCl}_{3}\right) \delta 14.8\left(\mathrm{CH}_{2} \mathrm{CH}_{3}\right)$, $17.8\left(\mathrm{CH}_{3}\right), 20.7\left(\mathrm{COCH}_{3}\right), 44.8(\mathrm{C}-7), 61.7\left(\mathrm{CH}_{2} \mathrm{CH}_{3}\right), 69.8(\mathrm{C}-4), 71.7(\mathrm{C}-6), 117.9(\mathrm{C}-2)$, $147.2(\mathrm{C}-3), 159.2\left(\mathrm{C}_{2}-\mathrm{CO}\right), 165.7(\mathrm{C}-8), 167,8\left(\mathrm{COCH}_{3}\right)$; MS (HR-ESI) m/z: $(\mathrm{M}+\mathrm{Na})^{+}$calcd for $\mathrm{C}_{12} \mathrm{H}_{15} \mathrm{NO}_{6} \mathrm{Na}: 292.0792$. Found: 292.0802 .

32. syrup; $[\alpha]_{\mathrm{D}}-87.84\left(\mathrm{c} 0.39, \mathrm{CH}_{2} \mathrm{Cl}_{2}\right)$; IR $\left(\mathrm{CH}_{2} \mathrm{Cl}_{2}\right) 1785,1766 \mathrm{~cm}^{-1} ;{ }^{1} \mathrm{H} \mathrm{NMR}\left(\mathrm{CDCl}_{3}\right): 1.35$ (t, 3H, J 7.1 Hz, $\mathrm{CH}_{2} \mathrm{CH}_{3}$ ), 1.47 (d, 3H, J 6.9 Hz, Me), 3.06 (dd, 1H, J 15.5, $0.9 \mathrm{~Hz}, \mathrm{H}_{\mathrm{a}}-7$ ), 3.26 (ddd, 1H, $J$ 15.5, 3.4, $\left.1.5 \mathrm{~Hz}, \mathrm{H}_{\mathrm{b}}-7\right), 3.29$ (dd, $\left.1 \mathrm{H}, J 13.9,1.5 \mathrm{~Hz}, \mathrm{H}_{\mathrm{a}}-2\right)$ ), 4.29 (2q, 2H, $J .1$ $\mathrm{Hz}, \mathrm{CH}_{2} \mathrm{CH}_{3}$ ), 4.65 (dd, 1H, J 14.0, $\left.0.8 \mathrm{~Hz}, \mathrm{H}_{\mathrm{b}}-2\right), 4.72$ (q, 1H, J 6.9 Hz, H-4), 5.23 (d, 1H, $3.0 \mathrm{~Hz}, \mathrm{H}-6) ;{ }^{13} \mathrm{C} \mathrm{NMR}\left(\mathrm{CDCl}_{3}\right) \delta 11.3,14.0,41.9(\mathrm{t}), 45.8(\mathrm{t}), 65.9(\mathrm{t}), 72.0,72.6,116.7(\mathrm{~s})$, 137.5 (s), 151.7 (s), 167.4 (s); MS (HR-LSIMS) m/z: (M+H) ${ }^{+}$calcd for $\mathrm{C}_{11} \mathrm{H}_{15} \mathrm{~N}_{2} \mathrm{O}_{5}$ : 255.09810 . Found: 255.09851.

33. syrup; IR $\left(\mathrm{CH}_{2} \mathrm{Cl}_{2}\right) 1785 \mathrm{~cm}^{-1} ;{ }^{1} \mathrm{H} \mathrm{NMR}\left(\mathrm{CDCl}_{3}\right)$ : 1.45 (d, 3H, J 6.9 Hz, Me), 2.15 (s, 3H, Ac), 3.06 (d, 1H, J $\left.15.5 \mathrm{~Hz}, \mathrm{H}_{\mathrm{a}}-7\right), 3.23$ (br d, 1H, J $\left.12.8 \mathrm{~Hz}, \mathrm{H}_{\mathrm{a}}-2\right), 3.26$ (ddd, 1H, J 15.4, 3.2, $\left.1.4 \mathrm{~Hz}, \mathrm{H}_{\mathrm{b}}-7\right), 4.65\left(\mathrm{~d}, 1 \mathrm{H}, J 14.1 \mathrm{~Hz}, \mathrm{H}_{\mathrm{b}}-2\right), 4.71$ (q, $\left.1 \mathrm{H}, J 6.9 \mathrm{~Hz}, \mathrm{H}-4\right), 5.23$ (d, 1H, J 3.1 Hz, $\mathrm{H}-6) ;{ }^{13} \mathrm{C}$ NMR $\left(\mathrm{CDCl}_{3}\right) \delta 11.4\left(\mathrm{CH}_{3}\right), 20.6,42.1,45.8(\mathrm{C}-7), 67.5(\mathrm{C}-3), 71.8(\mathrm{C}-4), 72.7$ (C-6), $116.7(\mathrm{CN}), 167.3(\mathrm{C}-8), 167.9\left(\mathrm{CH}_{3} \mathrm{CO}\right)$; MS (HR-LSIMS) m/z: $(\mathrm{M}+\mathrm{H})^{+}$calcd for $\mathrm{C}_{10} \mathrm{H}_{13} \mathrm{~N}_{2} \mathrm{O}_{4}$ : 225.08753. Found: 225.08799.

\section{References}

1. Cama, L.D.; Christensen, B.G. J. Am. Chem. Soc. 1974, 96, 7582.

2. Firestone, R.A.; Fahey, J. L.; Maciejewicz, N.S.; Patel, G.S.; Christensen, B.G. J. Med. Chem. 1977, 20, 551.

3. (a) Nagata, W.; Narisada, M.; Yoshida, T. In Chemistry and Biology of $\beta$-Lactam Antibiotics; Eds.; Morin, R. B.; Gorman, M. Academic Press: New York, 1982; Vol. 2, p 1. (b) Nagata, W. In Current Trends in Organic Synthesis; Eds.; Nozaki, H. Pergamon Press: Oxford, 1983; p 83.

4. Shibahara, S.; Okonogi, T.; Murai, Y.; Kudo, K.; Yoshida, T.; Kondo, S.; Christensen, B.G. J. Antibiot. 1988, 41, 1154.

5. Bełżecki, C.; Urbański, R.; Urbańczyk-Lipkowska, Z.; Chmielewski, M. Tetrahedron 1997, $53,14153$. 
6. Kałuża, Z.; Kazimierski, A.; Lewandowski, K.; Suwińska, K.; Szczęsna, B.; Chmielewski M. Tetrahedron 2003, 59, 5893.

7. (a) Kałuża, Z.; Park, H.-S. Synlett 1996, 895; Kałuża, Z.; Łysek, R. Tetrahedron: Asymmetry 1997, 8, 2553. (b) Kałuża, Z.; Łysek, R. Tetrahedron Lett. 1998, 39, 8349. (c) Kałuża, Z. Tetrahedron Lett. 1999, 40, 1025. (d) Kałuża, Z.; Furman, B.; Krajewski, P.; Chmielewski, M. Tetrahedron 2000, 56, 5553. (e) Borsuk, K.; Kazimierski, A.; Solecka, J.; UrbańczykLipkowska, Z.; Chmielewski, M. Carbohydr, Res. 2002, 337, 2005.

8. (a) House, H.O.; Kramar, V. J. Org. Chem. 1963, 28, 3362. (b) House, H.O.; Trost, B.M. J. Org. Chem. 1965, 30, 1341, 2502. (c) House, H.O.; Tefertiller, B.; Olmstead, H.D. J. Org. Chem. 1968, 33, 935. (d) House, H.O.; Bare, T.M. J. Org. Chem. 1968, 33, 943. (e) House, H.O.; Czuba, L. J.; Gall, M.; Olmstead, H. D. J. Org. Chem. 1969, 34, 2324. (f) House, H. O. Rec. Chem. Prog. 1967, 28, 99. (g) Huff, B. J. L.; Tuller, F. N.; Caine, D.J. J. Org. Chem. 1969, 34, 3070.

9. Fang, C.; Suganurma, K.; Suenume, H.; Sakai, K. J. Chem. Soc., Perkin Trans 1 1991, 6, 1549.

10. (a) Crumbie, R.L.; Nimitz, J.S.; Mosher, H.S. J. Org. Chem. 1982, 47, 4040. (b) Tsunokawa, Y.; Iwasaki, S.; Okuda, S. Tetrahedron Lett. 1992, 23, 2113.

11. (a) Immer, H.; Bagli, J.F. J. Org. Chem. 1968, 33, 2457. (b) Johnson, W. S.; Jensen, N. P.; Hooz, J.; Leopold, E. J. J. Am. Chem. Soc. 1968, 90, 5872.

12. Al-Awadi, N.; Taylor, R. J. Chem. Soc., Perkin Tran 2 1986, 1581.

13. Wang, C.; Kingsbury, C. A.; J. Org. Chem. 1975, 40, 3811.

14. Ghosez, L.; Marchand-Brynaert, J.; Vakemans, J.; Bogdan, S.; Cossement, E. Tetrahedron 1983, 39, 2493. 